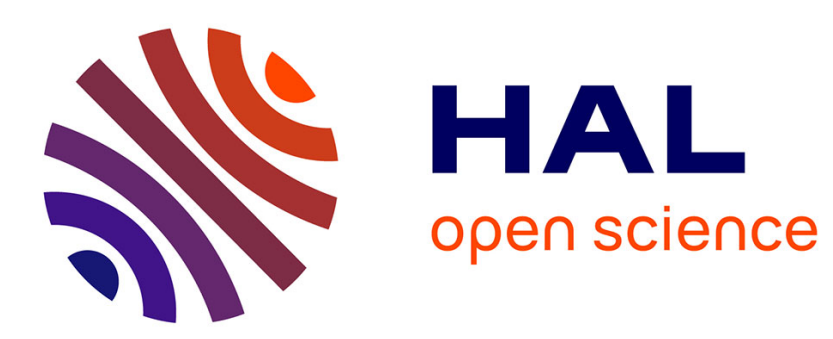

\title{
Structural Phase Transformation and Phonon Softening in Iron-Based Alloys
}

\author{
H. Herper, E. Hoffmann, P. Entel, W. Weber
}

\section{To cite this version:}

H. Herper, E. Hoffmann, P. Entel, W. Weber. Structural Phase Transformation and Phonon Softening in Iron-Based Alloys. Journal de Physique IV Proceedings, 1995, 05 (C8), pp.C8-293-C8-298. 10.1051/jp4:1995841 . jpa-00254090

\section{HAL Id: jpa-00254090 https://hal.science/jpa-00254090}

Submitted on 1 Jan 1995

HAL is a multi-disciplinary open access archive for the deposit and dissemination of scientific research documents, whether they are published or not. The documents may come from teaching and research institutions in France or abroad, or from public or private research centers.
L'archive ouverte pluridisciplinaire HAL, est destinée au dépôt et à la diffusion de documents scientifiques de niveau recherche, publiés ou non, émanant des établissements d'enseignement et de recherche français ou étrangers, des laboratoires publics ou privés. 


\title{
Structural Phase Transformation and Phonon Softening in Iron-Based Alloys
}

\author{
H.C. Herper, E. Hoffmann, P. Entel and W. Weber* \\ Theoretische Tieftemperaturphysik, Gerhard-Mercator-Universität, Gesamthochschule Duisburg, 47048 \\ Duisburg, Germany \\ * Theoretische Physik II, Universität Dortmund, 44221 Dortmund, Germany
}

\begin{abstract}
The interrelation of Invar and Martensite in iron-based alloys like $\mathrm{Fe}_{3} \mathrm{Ni}$ is discussed on the basis of $a b$ initio calculations for different crystal structures. Inelastic neutron scattering and ultrasonic experiments have shown that a soft transverse phonon mode in [110] direction acts as a precursor in the close packed fcc phase of these systems. Unfortunately there are no experimental results for phonon modes in the transformed bct phase. We have examined in detail the changes in the phonon dispersions of these systems by varying the ratio of the lattice constants along the Bain path. In recent calculations we have used the method of Varma and Weber in combination with the full potential LAPW (FLAPW) method to calculate frozen phonons for the tetragonal phase. Our new numerical results reproduce the experimental findings for the fcc phase and give an overview of the changes of the phonon energies when varying the $c / a$ ratio towards the bct structure. In particular we observe that phonon softening is caused by the long-range electron-phonon interaction in systems with magnetovolume instabilities, and vanishes after the martensitic transformation.
\end{abstract}

\section{INTRODUCTION}

$\mathrm{X}$-ray investigations and neutron scattering experiments have shown that transition metal alloys like $\mathrm{Fe}_{65} \mathrm{Ni}_{35}$ and $\mathrm{Fe}_{72} \mathrm{Pt}_{28}$ undergo a martensitic phase transition from the close packed high temperature fcc phase to the open ground state (bct) structure. Fig. 1 shows the experimental phase diagram for the $\mathrm{Fe}-\mathrm{Ni}$ system [1]. It can be seen that iron rich systems undergo a $\gamma$ - $\alpha$ transition and that the martensitic phase transformation overlaps with the Invar region.

In our work we have used the ordered $\mathrm{Fe}_{3} \mathrm{Ni}$ system as an approximation for the disordered $\mathrm{Fe}_{1-\mathrm{x}} \mathrm{Ni}_{\mathrm{x}}$ alloys with $25 \leq x \leq 35$. We study the total energy varying the $\mathrm{c} / \mathrm{a}$ ratio along the bain path with constant volume from bct to fcc (Fig. $2 \mathrm{~b}$ ). In addition to this we have calculated the phonon dispersion of both phases in order to study possible precursor effects of the martensitic transition on the phonon frequencies. Typical for the close packed structure is a well known soft $\mathrm{TA}_{1}$ phonon mode in the [110] direction. In this paper we want to show that this mode acts as a precursor for the $\gamma-\alpha$ transformation. In order to do this we calculate the phonon dispersion of fcc and bct $\mathrm{Fe}_{3} \mathrm{Ni}$. To day there exists not much experimental data about the tetragonal distorted phase of $\mathrm{Fe}_{65} \mathrm{Ni}_{35}$ because it is experimentally hardly accessible. For the fcc phase we made a semi ab initio investigation of the phonon frequencies $\omega(q)$ using the tight-binding scheme of Varma and Weber $[2,3]$. The bct system is more complex because of the just mentioned missing experimental information. We performed FLAPW calculations [4] by using the general gradient approximation (GGA) [5] which allow the evaluation of force constants for bct $\mathrm{Fe}_{3} \mathrm{Ni}$. 


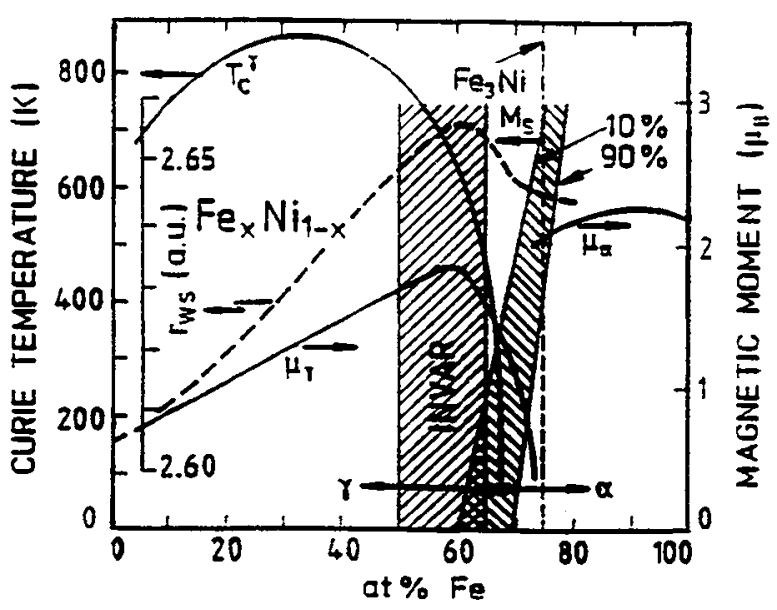

Fig. 1: Magnetic phase diagram of $\mathrm{Fe}_{\mathrm{x}} \mathrm{Ni}_{1-\mathrm{x}}$ based on experimental data [1]. Curie temperature $\mathrm{T}_{\mathrm{c}}$ in the $\gamma$ phase and the magnetic moment of the Fe atoms are given. The hatched areas mark the Invar region and the martensitic transformation range.

\section{METHOD}

\subsection{Electronic structure}

The calculation of the electron-phonon matrix elements with the procedure of Varma and Weber requires band-structure calculations. For $c / a=1.0(\mathrm{fcc})$ and 0.70711 (bct) this has been done with the ASW method in the framework of LDA. We replaced the bandstructures by a Slater-Koster tightbinding scheme in order to get information about the one particle states and the transfer integrals. For both cases, only bands crossing or touching the Fermi level have been taken into account because these are the most important ones for the electron-phonon matrix elements.

\subsection{Electron-phonon coupling}

The contribution of the electron-phonon interaction to the dynamical matrix $\mathcal{D}$ has been obtained within second order perturbation theory. The matrix can be written as $\mathcal{D}_{0}+\mathcal{D}_{2}$, with $\mathcal{D}_{0}$ containing all short range interaction terms. This part can be described by a force constant model. $\mathcal{D}_{2}$ reflects all long range forces and arises from the 2 nd order perturbation term

$$
\mathcal{D}_{2}\left(\kappa \alpha, \kappa^{\prime} \beta \mid \vec{q}\right)=-\sum_{\vec{k}, \vec{k}^{\prime}=\vec{k}+\vec{q}, \mu, \nu} \frac{f_{\vec{k}^{\prime} \nu}-f_{\vec{k}^{\prime} \mu}}{\epsilon_{\vec{k} \mu}-\epsilon_{\vec{k}^{\prime} \nu}} g_{\vec{k} \mu, \vec{k}^{\prime} \nu^{\prime}}^{\kappa \alpha} g_{\vec{k}^{\prime} \nu, \vec{k} \mu}^{\kappa^{\prime} \beta},
$$

with the electron-phonon matrix element $g_{\overrightarrow{k^{\prime}} \nu, \vec{k} \mu}^{\kappa^{\prime} \beta}$. It connects electronic eigenvectors with the distortion of the atoms from their equilibrium positions. $\mathcal{D}_{2}$ also contains the Fermi distribution and the electronic energies. From theoretical investigations we know that the $\mathrm{Fe}_{3} \mathrm{Ni}$ system is ferromagnetic for each ratio $c / a$, see for example Fig. 2a. In view of this fact we use an extended formalism for the electron-phonon coupling theory of Varma and Weber which allows to take magnetic order into account in a simplified manner [6].

\subsection{Force constants}

We have calculated the short range force constants for the fcc structure by using a Born-von Kármán model with the help of experimental data for elastic constants, phonon energies etc. Now for the 
tetragonal state we have to take the force constants from density functional theory (DFT) because of missing experimental data.
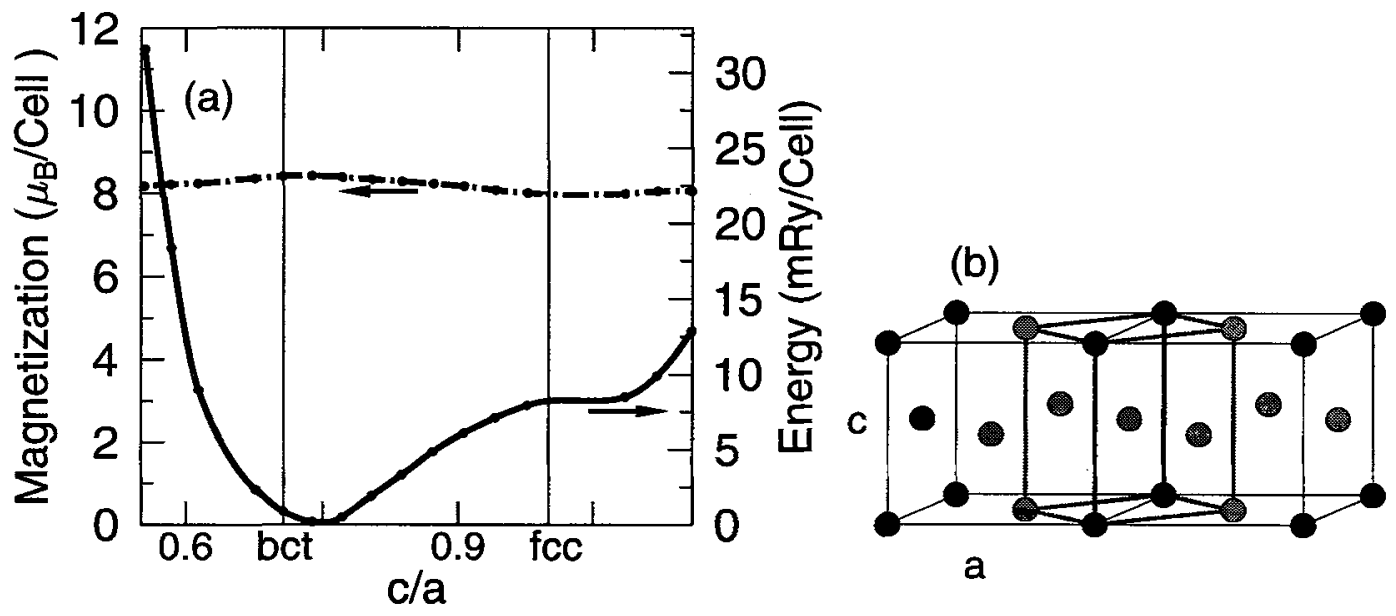

a

Fig. 2: (a) Total energy and magnetization of $\mathrm{Fe}_{3} \mathrm{Ni}$ for different $\mathrm{c} / \mathrm{a}$ ratios. These results were obtained from FLAPW calculations corrected by the generalized gradient approximation (GGA)[5]. These calculations were done for constant volume of the bct unit cell, shown in (b).

In our case we make use of the FLAPW formalism calculating the total energy of different frozen phonons. This method is very time and memory consuming and to day we have only calculated 8 different distortions in order to get the nearest neighbour terms. The force constants are defined as 2nd derivative of the distortion energy.

\section{RESULTS AND DISCUSSION}

$\mathrm{Fe}-\mathrm{Ni}$ alloys undergo a martensitic phase tranformation in a range of 70 to 100 at\% iron, where 70 at\% $\mathrm{Fe}$ is close in concentration to the Invar region. This makes it difficult to distinguish whether changes in the phonon dispersion are caused by the Invar effect or by the onset of the structural phase transition.

We first calculated the total energy of $\mathrm{Fe}_{3} \mathrm{Ni}$ varying the c/a ratio along the Bain path shown in Fig. $2 \mathrm{~b}$ for constant volume of the unit cell. The bct magnetic moment compares well with experimental findings in Fig. 1 and agrees with our former ASW calculations [7]. For calculated bct $\mathrm{Fe}_{3} \mathrm{Ni}$ we obtained a magnetic moment of about $8.3 \mu_{B}$ per cell, see Fig. 2a. The Wigner-Seitz radius is about 2.65 a.u. which is larger than the experimental one. This can be explained by the fact that in measurements the magnetic moment starts to become unstable with the beginning of the martensitic transformation. It is also obvious from Fig. 2a that for $\mathrm{T}=0$ the system favours the bct structure and that for $c / a=1.0$ we have a saddle point which lies at higher energy.

We have determined the phonon dispersion for both phases. Using the tight binding method of Varma and Weber, we were able to reproduce the experimental findings [8] for the fcc state quite well [6], which can be seen in Fig. 3a. The softening of the $\mathrm{TA}_{1}[110]$ phonon mode compares well with the neutron scattering data from Endoh and Noda. For bct $\mathrm{Fe}_{3} \mathrm{Ni}$ we took the short-range force constants from FLAPW calculations while the long-range forces arising from the electron-phonon interactions were obtained within the framework of Varma's theory. The resulting phonon spectrum is shown in Fig. 4a. We have calculated it for all high symmetry lines and points of the simple tetragonal Brillouin zone, see Fig. 4b: The bct phase as compared to the fcc structure has no soft phonons. The degeneration is smaller than in the fcc case because of the broken symmetry in [111] 
direction, see for example the dispersion along the $\Gamma-\mathrm{X}$ direction. Besides this observation both spectra are very similar. For the tetragonal distorted phase, the maximum phonon energy is about $2 \mathrm{meV}$ smaller than for the close packed structure. In order to search for a connection between

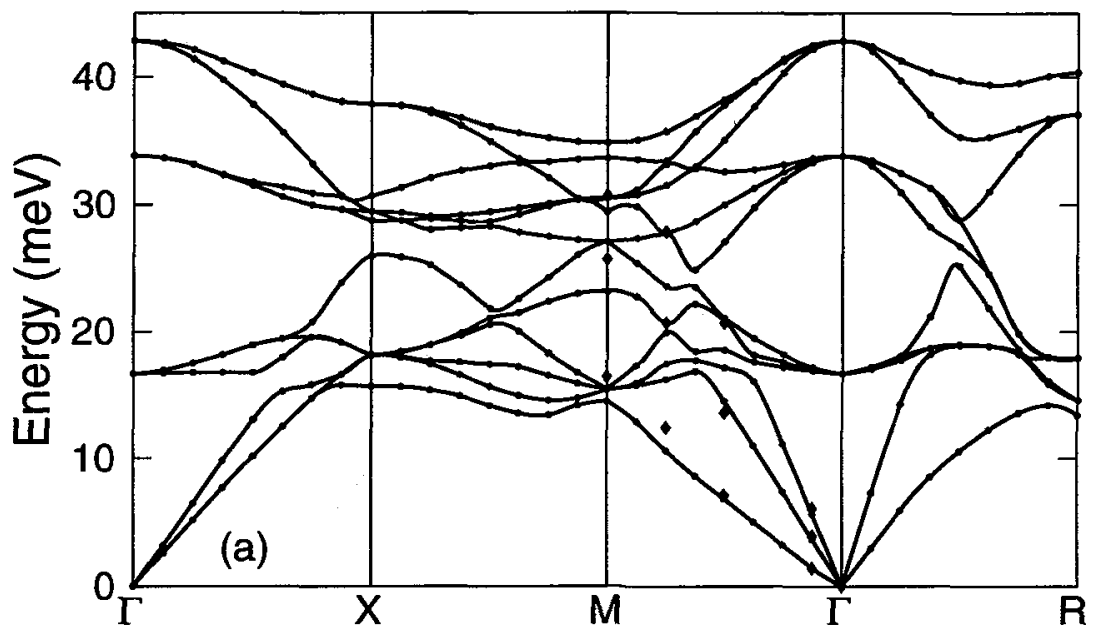

(b)

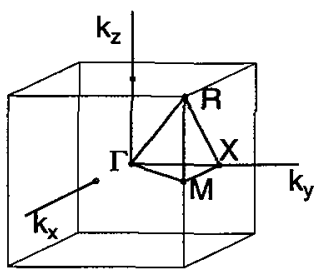

Fig. 3: (a) Renormalized phonon dispersion for fcc $\mathrm{Fe}_{3} \mathrm{Ni}$. Full lines connect the calculated points with 3rd order splines fit. Diamonds represent experimental results [8]. We calculated the spectrum along the lines of high symmetry marked in the simple cubic BZ, shown in (b).
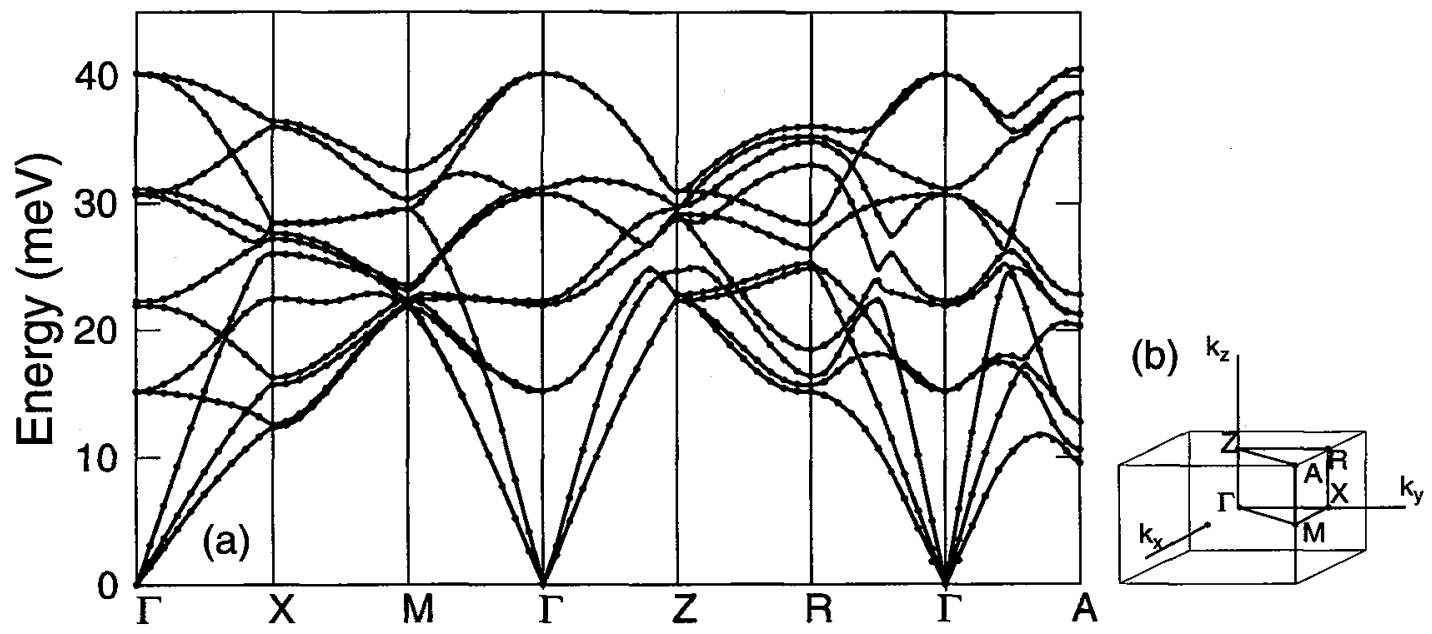

Fig. 4: (a) Phonon frequencies for bct $\mathrm{Fe}_{3} \mathrm{Ni}$. We used selfconsistently determined force constants for the short range forces and the procedure of Varma and Weber, in order to obtain the long-range contributions.

anomalous lattice vibrations and the martensitic phase transformation, the phase stability has been tested by changing the electron-phonon coupling strength. If we cool down fcc $\mathrm{Fe}_{3} \mathrm{Ni}$ the density of states at the Fermi energy $\mathrm{E}_{\mathrm{F}}$ is increased and the magnetic moment increases by moving the high-temperature low-spin state towards $\mathrm{E}_{\mathrm{F}}$, this can be seen in Fig. 5a. But larger density of states causes a larger electron-phonon coupling. Therefore, we simulated the temperature evolution by increasing the electron-phonon matrix elements without varying the structural parameters. 
Increasing gradually the coupling constants the eigenvalues are decreased but the softening of the $\mathrm{TA}_{1}[110]$ mode is now much stronger. For $\mathcal{D}_{2}$ about 1.71 times bigger than the original electron-
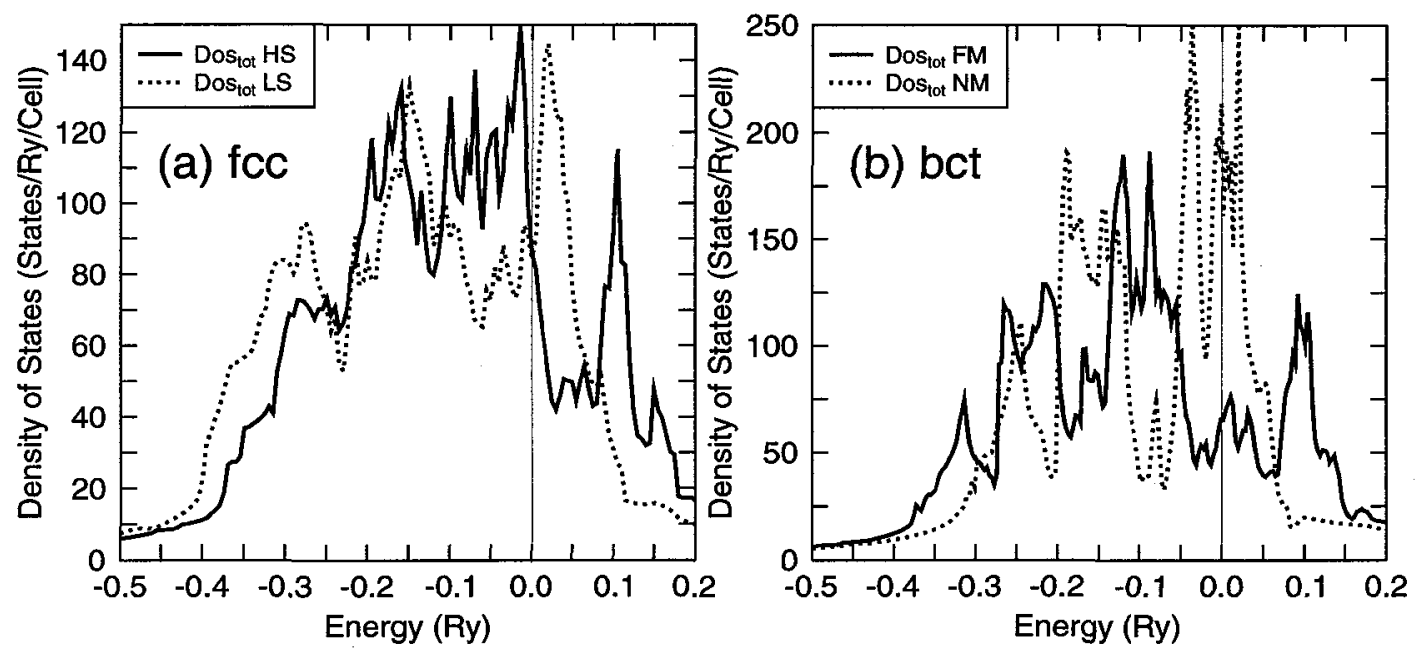

Fig. 5: (a) Total electronic density of states per unit cell for $\mathrm{Fe}_{3} \mathrm{Ni}$ in the fcc and (b) bct phase. The dotted curves mark the low-spin state in the fcc phase and the nonmagnetice case in the bct structure. The ferromagnetic high-spin state in (a) and (b) is characterized by the full line.

phonon coupling, negative phonon frequencies appear in the spectrum and the system is not longer stable. Fig. 6 reflects the change of this transversal mode for different coupling strengths. From this we may conclude that for $\mathrm{T} \rightarrow 0$, the fcc state is unfavourable and the soft phonon $\mathrm{TA}_{1}$ mode acts as a precursor of the structural $\gamma-\alpha$ phase transformation.

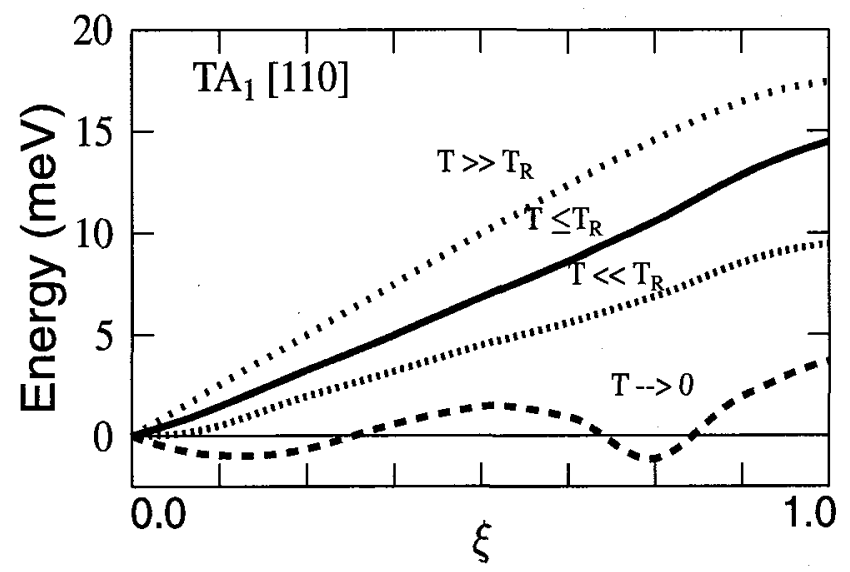

Fig. 6: Phonon energies of the $\mathrm{TA}_{1}$ mode in [110] direction for different coupling strengths. $\mathrm{T}_{\mathrm{R}}$ is the room temperature.

In contrast to these observations, the phonon dispersion for bct $\mathrm{Fe}_{3} \mathrm{Ni}$ itself contains no anomalous curvatures, at $\mathrm{T}=0$ it is stable. This confirms the existence of a bct ground state for the $\mathrm{Fe}_{3} \mathrm{Ni}$ system. But what will happen to the bct state when we go to finite temperatures? We made a similar experiment as for fcc $\mathrm{Fe}_{3} \mathrm{Ni}$ by increasing the strength of the electron-phonon interaction. Also in this case a similar density of states argument is important, however, this time the magnetization 
decreases, see Fig. 5b. The whole phonon dispersion moves to smaller energies as expected but without showing a special softening. Fig. 7 shows, for example, results for the $\Gamma$-M direction with a 1.44 times stronger electron-phonon matrix than the calculated $\mathcal{D}_{2}$.

The extremely strong softening of zone boundary phonons, see for example the $\mathrm{X}$ point in Fig. 7,

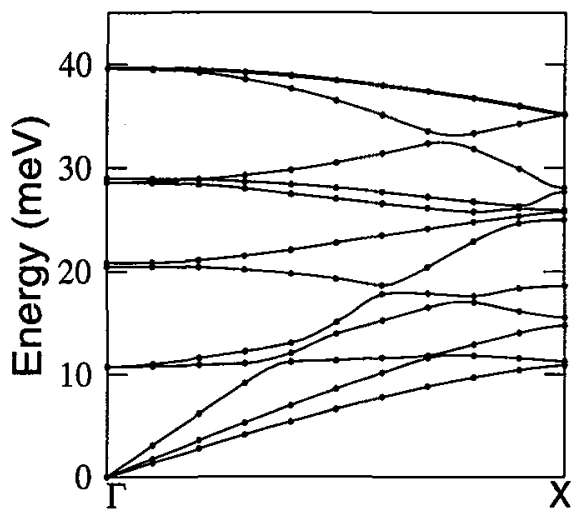

Fig. 7: Phonon dispersion in [100] direction for bct $\mathrm{Fe}_{3} \mathrm{Ni}$ with $\mathcal{D}_{2}$ 1.44 times stronger than the calculated electron-phonon matrix.

may be an artifact of our calculations because we could take into account only first nearest neighbours for the evaluation of the force constants. Nevertheless, one may see that the bct structure becomes less stable at finite temperatures.

In this paper we have tried to show that the softening of the close packed fcc phase of $\mathrm{Fe}_{3} \mathrm{Ni}$ is a precursor of the martensitic phase transition. This tendency has been obtained from the different behaviour of the electron-phonon interaction in the fcc and bct state. Whether this important aspect found for the $\mathrm{Fe}-\mathrm{Ni}$ system also plays a decisive role in $\mathrm{Fe}-\mathrm{Pd}$ and $\mathrm{Fe}-\mathrm{Pt}$ remains to be checked.

\section{ACKNOWLEDGEMENTS}

We would like to thank Prof. Dr. E.F. Wassermann and his collaborators for helpful discussions.

\section{References}

[1] Wassermann E.F., Ferromagn. Mat., Vol. V (North-Holland, Amsterdam, 1990), pp. 237-322.

[2] Varma C.M., Weber W., Phys Rev. Lett. 39 (1977) 1094-1098.

[3] Varma C.M., Blount E.I., Vashishta P. and Weber W., Phys Rev. B 19 (1979) 6130-6141.

[4] Blaha P., Schwarz K., Soratin P., and Trikey S.B., Comput. Phys. Commun. 59, (1990) 399-415.

[5] Perdew J.P., Chevary J.A., Vosko S.H., Jackson K.A., Pederson M.R. Singh D.J., and Fiolhais C., Phys Rev. B 46, (1992) 6671-6687.

[6] Herper H.C., Entel P., Weber W., "Electron-Phonon Interaction and Phonon Softening in Systems with Magnetovolume Instabilities", ESOMAT'94, Barcelona, 14-16 September 1994, A. Planes, J, Ortín and L. Mañosa (Journal de Physique IV, Colloque C2, Les Editions de Physique, France, 1995), pp. 129-134.

[7] Hoffmann E., Herper H., Entel P., Mishra S.G., Mohn P. and, Schwarz K., Phys. Rev. B 47 (1993) 5589-5596.

[8] Endoh Y., Noda Y., Ishikawa Y., Solid State Commun. 23 (1977) 951-953. 